\title{
Teamführung
}

\section{Mit wenigen Bausteinen zu mehr Effizienz}

\author{
Studien zufolge leistet ein gut eingespieltes Praxisteam einen \\ erheblichen Beitrag zum Erfolg einer Arztpraxis. Gut eingespielt ist ein \\ Team aber nur, wenn die Führungsspitze den notwendigen Rahmen \\ setzt. Das Problem vieler Ärzte: Sie sind auf diese Aufgabe zu Beginn \\ ihrer Tätigkeit meist nicht vorbereitet. Doch das lässt sich nachholen.
}

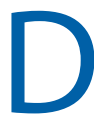
er häufigste Fehler steckt bereits in der Annahme, dass die Praxismitarbeiter die gerechte Verteilung von Aufgaben und Konflikte im Team „wie Erwachsene untereinander selbst regeln“. Bei Konflikten ist es zum Beispiel aber besonders wichtig, sich als Vorgesetzter frühzeitig einzuschalten. Streit im Praxisteam kann schnell eskalieren und zu einer Frontenbildung oder gar Spaltung des Teams führen. Zusätzlich besteht das Risiko, dass Patienten solche Streitigkeiten mitbekommen und diese negativen Beobachtungen im Bekanntenkreis weitererzählen.

\section{Leistung sichtbar machen}

Viele Ärzte ärgern sich auch über das mangelnde Engagement einzelner Mitarbeiter in Teambesprechungen. Wenn Menschen in einer Gruppe an einem gemeinsamen Ziel arbeiten, reduziert sich jedoch häufig die Leistung des Einzelnen. Der Effekt tritt immer dann ein, wenn unklar ist, wie viel jeder zur Gesamtleistung beiträgt. Dieses Zurücklehnen in der "sozialen Hängematte“ ist normal. Es ist kein Ausdruck von Unwillen. Gegensteuern lässt sich, indem neben Teamaufgaben auch individuelle Aufgaben verteilt werden, welche die Leistung des Einzelnen eben doch sichtbar machen. Wenn zum Beispiel als gemeinsames Praxisprojekt geplant ist, die Patientenzufriedenheit über verbesserten Service und patientenorientierte Kommunikation zu erhöhen, dann sollten zunächst einzelne Aspekte wie Telefonservice,
Wartezeiten oder Kommunikation mit anspruchsvollen Patienten an einzelne Mitarbeiter gegeben werden. Erst im Anschluss daran wird alles mit dem gesamten Praxisteam weiterentwickelt.

\section{Der ideale Mitarbeiter - ein Mythos?}

Einen absolut idealen Mitarbeiter gibt es wohl nicht, aber es gibt die ideale Besetzung für einen bestimmten Arbeitsplatz. Bei der Besetzung einer Stelle sollte daher darauf geachtet werden, welche Stärken und Talente der Mitarbeiter mitbringt. Und, an welchem Arbeitsplatz er diese am besten einsetzen kann. Demgegenüber wird der Einsatz nach defizitorientierten Gesichtspunkten, nach dem Motto „Das müssen Sie jetzt ganz besonders lernen“, eher einen negativen Effekt haben. Der entsprechende Mitarbeiter wird in diesem Bereich einfach nie so gut sein wie die Kollegen mit mehr Talent; die Fehlerhäufigkeit steigt, und Demotivation ist die Folge. Ein kontinuierliches Rotieren, wie es in vielen Praxen üblich ist, sollte daher gut überlegt sein. Denn dabei arbeiten Mitarbeiter immer wieder eine ziemlich lange Zeit auf Positionen, in denen sie eventuell talentfrei sind.

\section{Neue Mitarbeiter - was zählt?}

Die Praxismitarbeiter sind die wichtigste "Software" einer Praxis. Etwa 70\% des Eindrucks, den ein Patient aus der Praxis mitnimmt, resultiert nicht aus der Behandlung durch den Arzt, sondern aus dem Engagement der Mitarbeiter. Beim
Bewerbungsgespräch lassen sich Ärzte häufig besonders durch die fachliche Qualifikation eines Bewerbers beeindrucken. Weitaus weniger wird ein Blick auf die persönliche Eignung geworfen, und noch weniger wird diese systematisch in das Bewerbungsgespräch miteinbezogen. Wenn ein Bewerber als Person nicht zu einem Praxis-team passt, kann dies später zu erheblicher Unzufriedenheit und sogar zur Kündigung führen. Schon im Bewerbungsgespräch sollten deshalb Schlüsselqualifikationen wie Teamfähigkeit, Flexibilität, Kommunikations- und Kontaktfähigkeit abgefragt werden. Dazu können etwa einfache Situationen aus dem Praxisalltag als Fallbeispiele („Wie würden Sie reagieren, wenn ...") besprochen werden. Es könnte aber bei einem zweiten Gespräch auch der Ersthelfer mit dabei sein.

\section{Eigenen „Personaler" einsetzen} Zusammenfassend kann festgehalten werden, dass sich die fachliche Eignung eines Mitarbeiters im Nachhinein meist verbessern lässt, die soziale Kompetenz und Persönlichkeit dagegen kaum. Eine adäquate Teamführung und -entwicklung im Praxisalltag ohne fundiertes Hintergrundwissen gestaltet sich für viele Praxisinhaber naturgemäß sehr schwierig, da das Hauptaugenmerk auf der Behandlung von Patienten liegt. Um den Praxisinhaber zu entlasten, kann dieser wichtige Aufgabenbereich daher durchaus auf einen Praxismanager oder Ersthelfer übertragen werden. Dieser wäre dann aber vorab zu schulen.

\section{Dr. Andrea Schuhmacher}

Beraterin, Coach und Trainerin für

Personalentwicklung bei Dr. Schuhmacher

Consulting \& Training GmbH, Köln.

Referentin für Unternehmens-

kommunikation, Presse und PR bei

Frielingsdorf Consult GmbH, Köln 